\title{
ERYTHROCYTOSIS IN THIAMINE DEFICIENT RATS
}

\author{
Reiko HOBARA and Hajime YASUHARA \\ Department of Pharmacology, School of Medicine, Showa University. \\ 1-5-8 Hatanodai, Shinagawa-ku, Tokyo 142, Japan
}

Accepted July 22, 1981

\begin{abstract}
A thiamine ( $T$ ) deficient state in rats was produced by feeding the rats a $T$ deficient diet (TDD). At the stage of 13 days (TDD13 group), the number of red blood cells (RBC) and white blood cells (WBC), hematocrit $(\mathrm{Ht})$ and hemoglobin $(\mathrm{Hb})$ values decreased. On the other hand, after 30 days on the TDD (TDD30 group), the number of RBC was $819 \times 10^{4} /$ $\mathrm{mm}^{3}$ as against $631 \times 10^{4} / \mathrm{mm}^{3}$ in the normal control group ( $\mathrm{NC}$ group). Ht and $\mathrm{Hb}$ values also increased in the TDD30 group. These changes observed in the TDD30 group were significantly different from findings in the equal weight control group (EWC group) or in the pair fed control group (PFC group). The number of reticulocytes increased, the levels of 2.3-diphosphoglycerate (2,3-DPG) of RBC decreased and plasma erythropoietin levels increased in the TDD30 group. T levels of blood in the TDD13 group were $62(39-79) \mathrm{ng} / \mathrm{ml}$ as against 275 (196-412) $\mathrm{ng} / \mathrm{ml}$ in the $\mathrm{NC}$ group. T levels of blood in the TDD30 group were $102(17-365) \mathrm{ng} / \mathrm{ml}$, and widely varied. Decrease in 2.3-DPG produces an increase in $\mathrm{O}_{2}$ affinity to $\mathrm{Hb}$, and hypoxia is induced in the peripheral tissues. Furthermore these conditions stimulate erythropoietin production and finally the number of RBC increases. T deficiency produces anemia at an early stage and absolute erythrocytosis occurs at the late stage of $T$ deficiency. The increased osmotic resistance of $\mathrm{RBC}$ in hypotonic solution was also observed in the TDD30 group. This increase in osmotic resistance correlated with the decrease in cholesterol and phospholipid levels in the membrane of RBC.
\end{abstract}

Thiamine (T) plays an important role on glycolysis (1) which is necessary to perform the normal function and viability of RBC. $T$-responsive megaloblastic anemia has been reported (2,3). In an attempt to clarify the relationship hematogenesis, $\mathrm{RBC}$ function and $T$, we carried out various hematological tests on rats maintained on diets deficients in T. Our findings are reported herein.

\section{MATERIALS AND METHODS}

1. Animals and diet preparation: Male Sprague-Dawley rats weighing about $60 \mathrm{~g}$ were caged individually and fed a regular diet (RD) (Oriental Kobo Co. Ltd. which

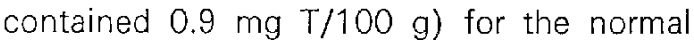
control group (NC group), a T deficient diet (TDD) for the TDD group (TDD group). and TDD plus $2.4 \mathrm{mg} \mathrm{T/100} \mathrm{g}$ for the pair fed control group (PFC group). The animals were fed a RD. of which weight was equally adjusted to that of the TDD group, for the equal weight control group (EWC group). The intake of water in the EWC group was also restricted as equal as in the TDD group. Various examinations were done at 13 days and 30 days fed a each diet. Rats were fasted for $18 \mathrm{hr}$. and blood sample was collected from the vena cava in a heparinized syringe. 
2. Hematological examinations and $T$ measurement: $\mathrm{RBC}$ and $\mathrm{WBC}$ were counted by Thoma's method after adequate dilution. Hematocrit $(\mathrm{Ht})$ value was measured by ultracentrifugal system using capillary. Hemoglobin $(\mathrm{Hb})$ concentration was assayed by Unikit hemoglobin-A (Chugai-Seiyaku Co. Ltd.). Reticulocytes were counted after dyeing by $0.5 \%$ brillant cresyl blue. 2,3-DPG value was assayed by Sigma's 2,3-DPG kit. Plasma erythropoietin levels were assayed by immunological method (Lack Laboratory Co. Ltd.). Plasma was collected about $1 \mathrm{ml}$ from each group for the assay of erythropoietin. T levels of blood and liver were assay by the biological method using Lactobacillus viridescens (4). Osmotic resistance of $\mathrm{RBC}$ was measured in various $\mathrm{NaCl}$ concentrations. One drop of blood was added into $\mathrm{NaCl}$ solution of various concentrations and incubated for $1 \mathrm{hr}$ at $37^{\circ} \mathrm{C}$, and centrifuged for $10 \mathrm{~min}$ at $3.000 \mathrm{rpm}$, and optical density of the supernatant was measured at $540 \mathrm{~nm}$. The values were expressed as the percent to the optical density of $100 \%$ hemolysis. Lipids of RBC membrane were extracted by Bragdon's method (5). Cholesterol was assayed by Zak's method (6), and phospholipid was assayed by Fiske-Subbarow's method (7).

\section{RESULTS}

1. Body weight gain and general symptoms: Body weight gain in the TDD group decreased from about 10 days after feeding a TDD, and at 30 days it was reduced to about $40 \%$ of the NC group. Body weight gain in the PFC group was same as that in the NC group (Fig. 1). In the TDD group, some neurological symptoms as abnormal gait were observed at about 25 days, and bleeding in left testis or both testes was found in some rats of this group at 30 days. but these findings were rarely seen in the EWC group. Congestion in mesenteric vein was observed in the TDD30 group.

2. Changes of blood components: The number of RBC decreased to $475 \times 10^{4} / \mathrm{mm}^{3}$ in the TDD13 group, and increased to $819 \times 10^{4} / \mathrm{mm}^{3}$ in the TDD30 group as

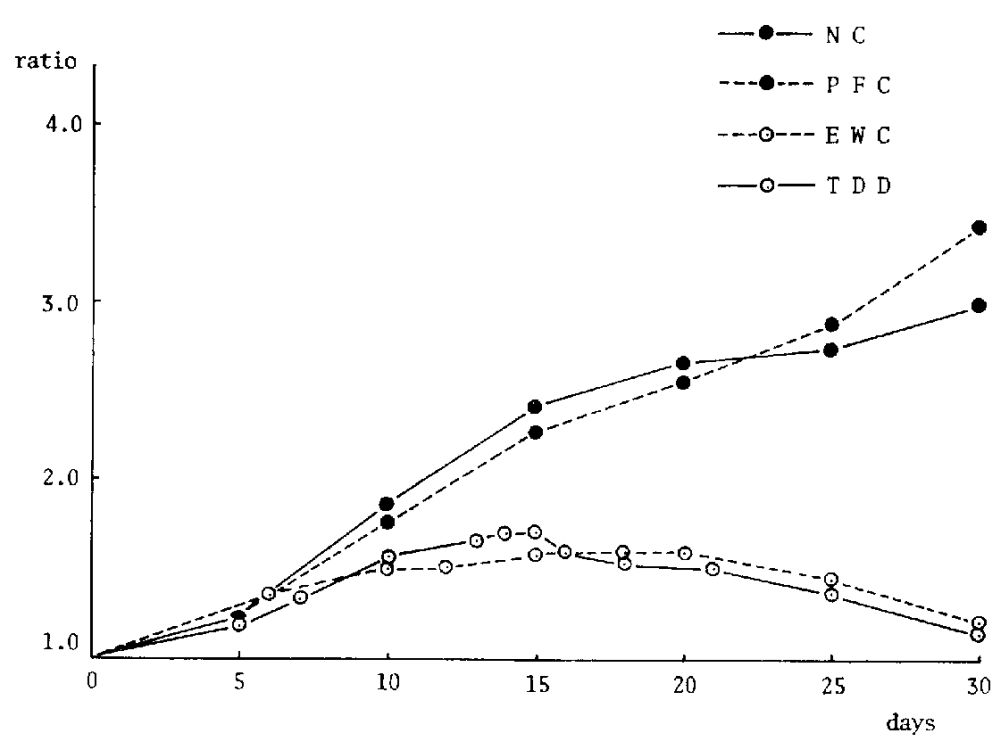

Fig. 1. Body weight gain. NC: normal control group, PFC: pair fed control group. EWC: equal weight control group, TDD: thiamine deficient diet group. 
against $631 \times 10^{4} / \mathrm{mm}^{3}$ in the NC group. EWC and TDD30 group as against $5,850 / \mathrm{mm}^{3}$ This change was significantly different from in the NC group (Fig. 3). $\mathrm{Ht}$ and $\mathrm{Hb}$ values in the EWC group. (Fig. 2). The number of the TDD30 group were $48.5 \%$ and $15.8 \mathrm{~g} / \mathrm{dl}$ WBC decreased to about $4,000 / \mathrm{mm}^{3}$ in the as against $40.5 \%$ and $12.5 \mathrm{~g} / \mathrm{dl}$ in the NC

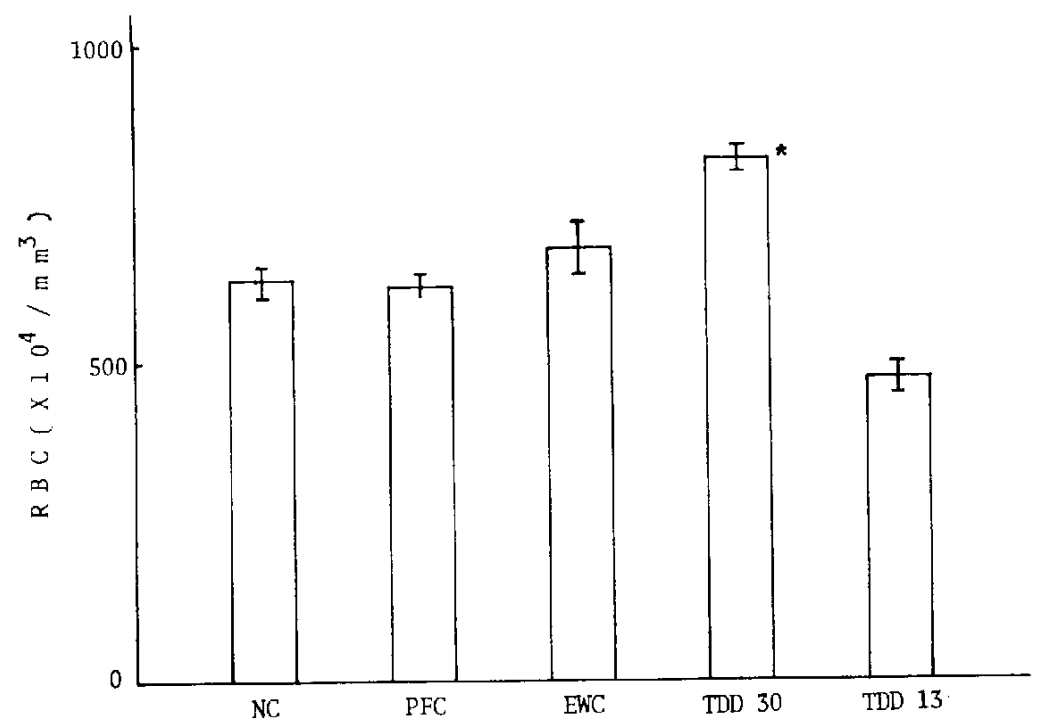

Fig. 2. Numbers of red blood cells (RBC) in TDD group compared with those in NC group. PFC group and EWC group. TDD 30: rats fed on TDD for 30 days. TDD13: rats fed on TDD for 13 days. Abbreviations are the same as Fig. 1. ${ }^{*} p<0.001$ corresponding NC group and $p<0.01$ corresponding EWC group.

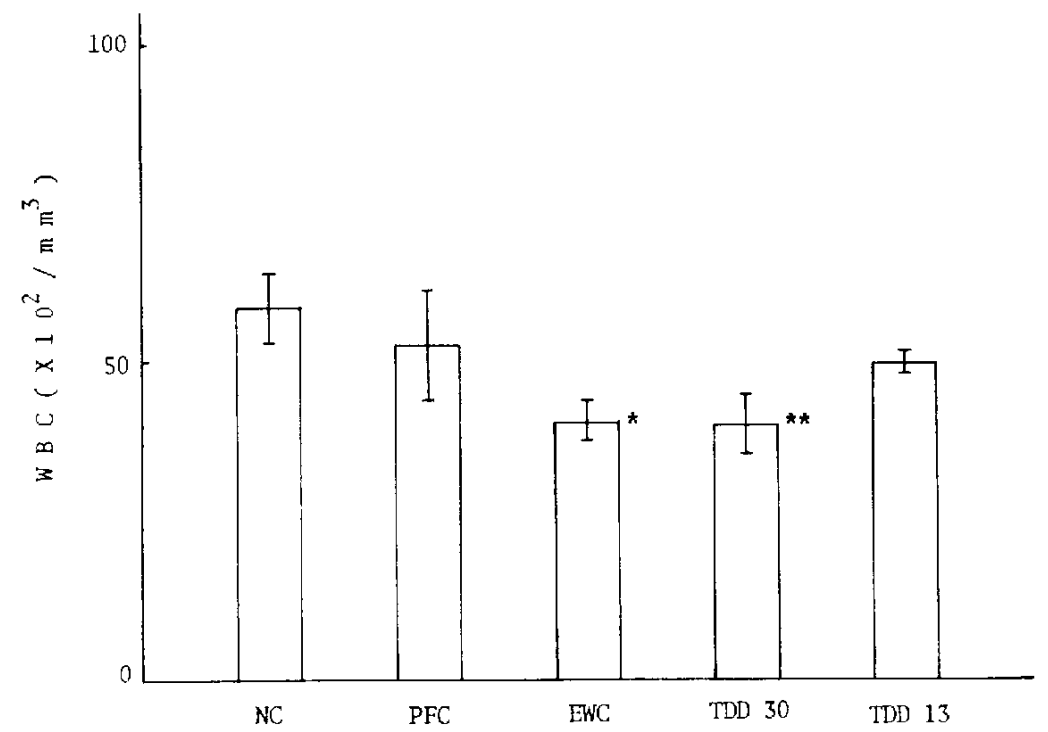

Fig. 3. Numbers of white blood cells (WBC) in TDD group compared with those in NC group. PFC group and EWC group. Abbreviations are the same as Fig. 2. "p<0.05 corresponding NC group, ${ }^{*} p<0.02$ corresponding NC group. 
group, respectively, and these increased against $62.0 \mu^{3}$ in the NC group. Mean significantly rather than those in the EWC corpuscular hemoglobin concentration group (Fig. 4 and Fig. 5). Mean corpuscular (MCHC) increased slightly in the TDD and volume (MCV) was $78.5 \mu^{3}$ in the TDD13 EWC group (Fig. 6). 2,3-DPG, which group, and $58.0 \mu^{3}$ in the TDD30 group as mediates the binding ability of Hb to oxygen

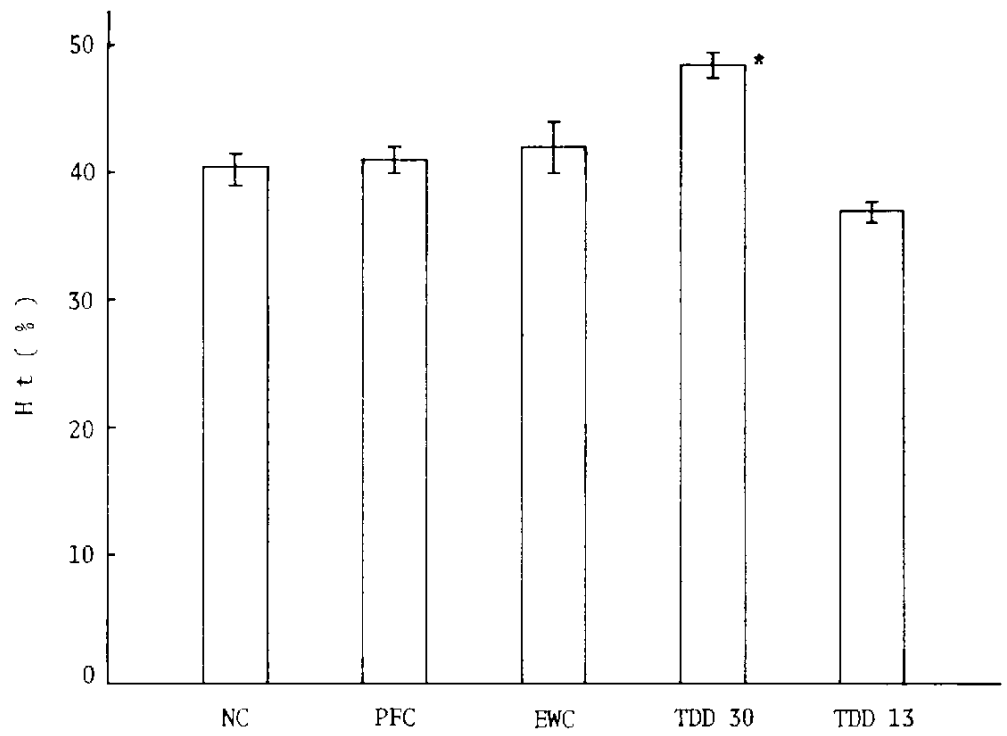

Fig. 4. Hematocrit values (Ht). Abbreviations are the same as Fig. 2. "p<0.001 corresponding $N C$ group and $p<0.01$ corresponding EWC group.

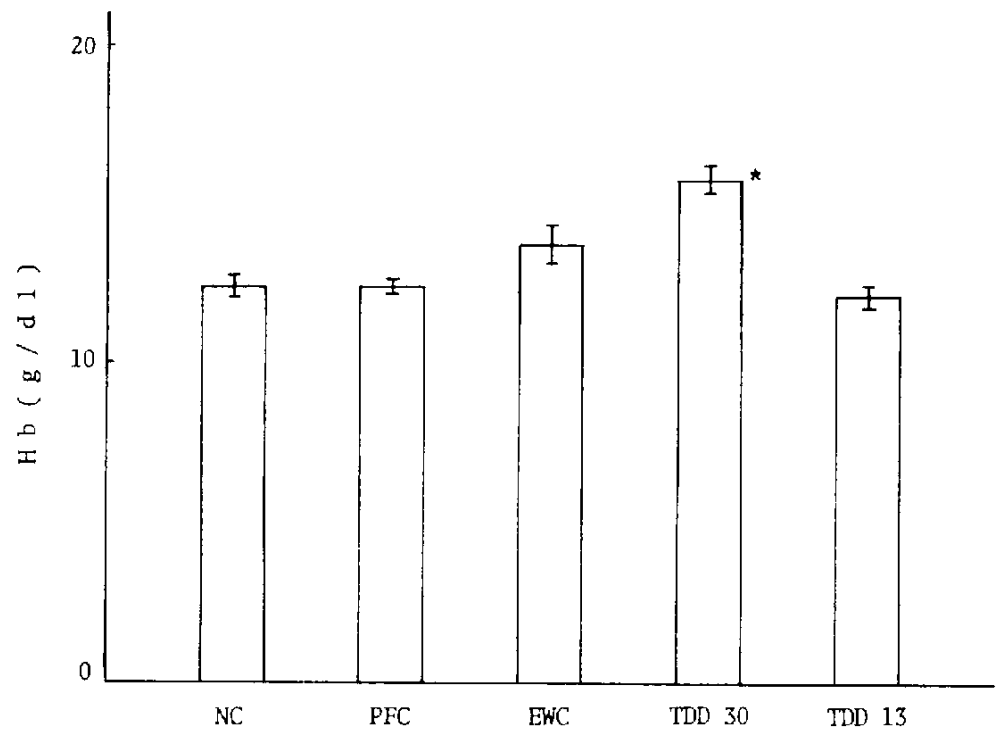

Fig. 5. Hemoglobin concentrations $(\mathrm{Hb})$. Abbreviations are the same as Fig. 2. " $p<0.001$ corresponding $\mathrm{NC}$ group and $p<0.02$ corresponding EWC group. 
$\left(\mathrm{O}_{2}\right)$, was $3.57 \mu \mathrm{M} / \mathrm{m} / \mathrm{RBC}$ in the TDD30 group, and were infrequently detected in the group. 2.3-DPG levels in the TDD30 group TDD30 group, but some cases in the TDD30 was markedly reduced as against $7.93 \mu \mathrm{M} /$ group, the number of them was about $61 \%$ mIRBC in the NC group (Fig. 7). Re- as against $30 \%$ in the NC group. Plasma ticulocytes were not detected in the EWC erythropoietin levels were 45 millim-

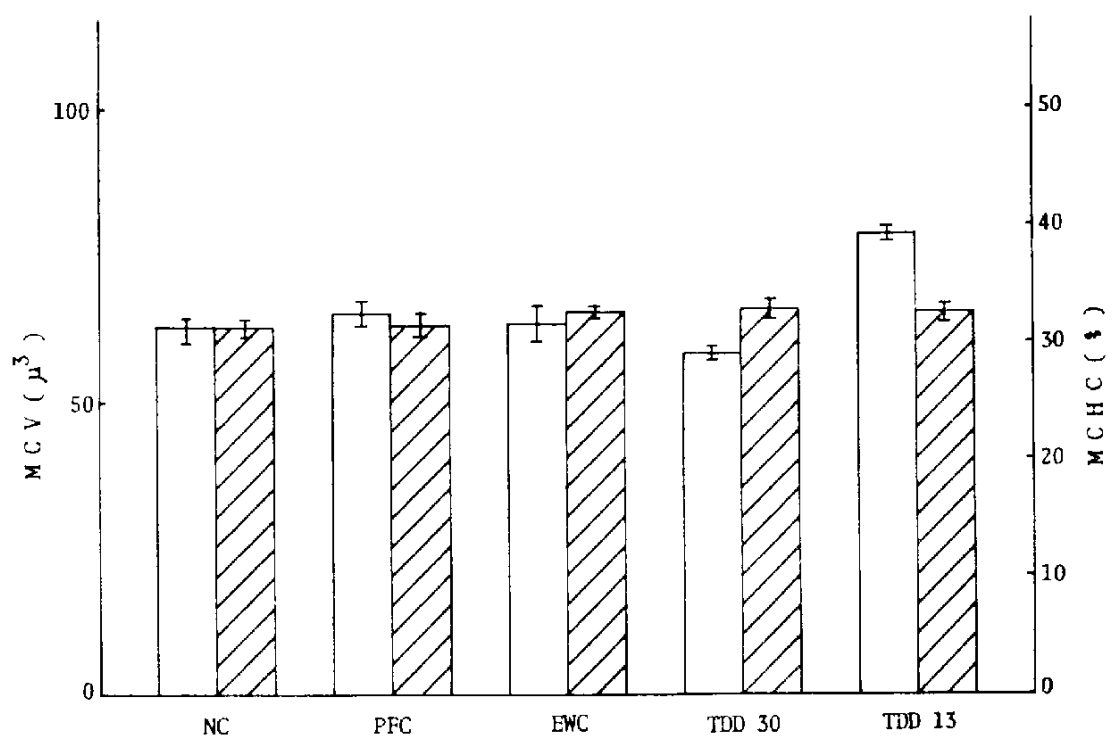

Fig. 6. Mean corpuscular volume (MCV) and mean corpuscular hemoglobin concentration (MCHC). Abbreviations are the same as Fig. 2.

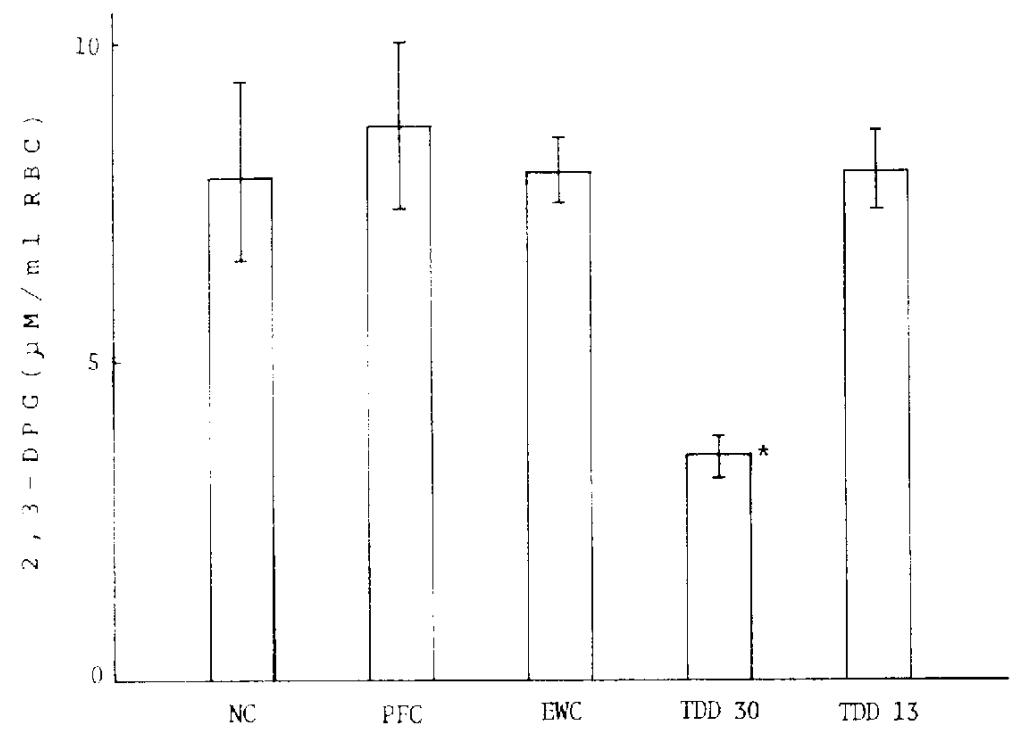

Fig. 7. Levels of 2,3-diphosphoglycerate (2,3-DPG). Abbreviations are the same as Fig. 2. " $p<0.001$ corresponding NC group and EWC group. 
munochemical U/ml in the TDD30 group as the TDD13 group, and $102 \mathrm{ng} / \mathrm{ml}$ in the against 15 millimmunochemical $\mathrm{U} / \mathrm{ml}$ in the TDD 30 group as against $275 \mathrm{ng} / \mathrm{ml}$ in the NC group. $N C$ group, but $T$ levels at 30 days varied

3. T levels of blood and liver: $T$ levels of widely from 17 to $365 \mathrm{ng} / \mathrm{ml}$. T levels in the blood decreased markedly to $62 \mathrm{ng} / \mathrm{ml}$ in PFC and EWC group were same as that in

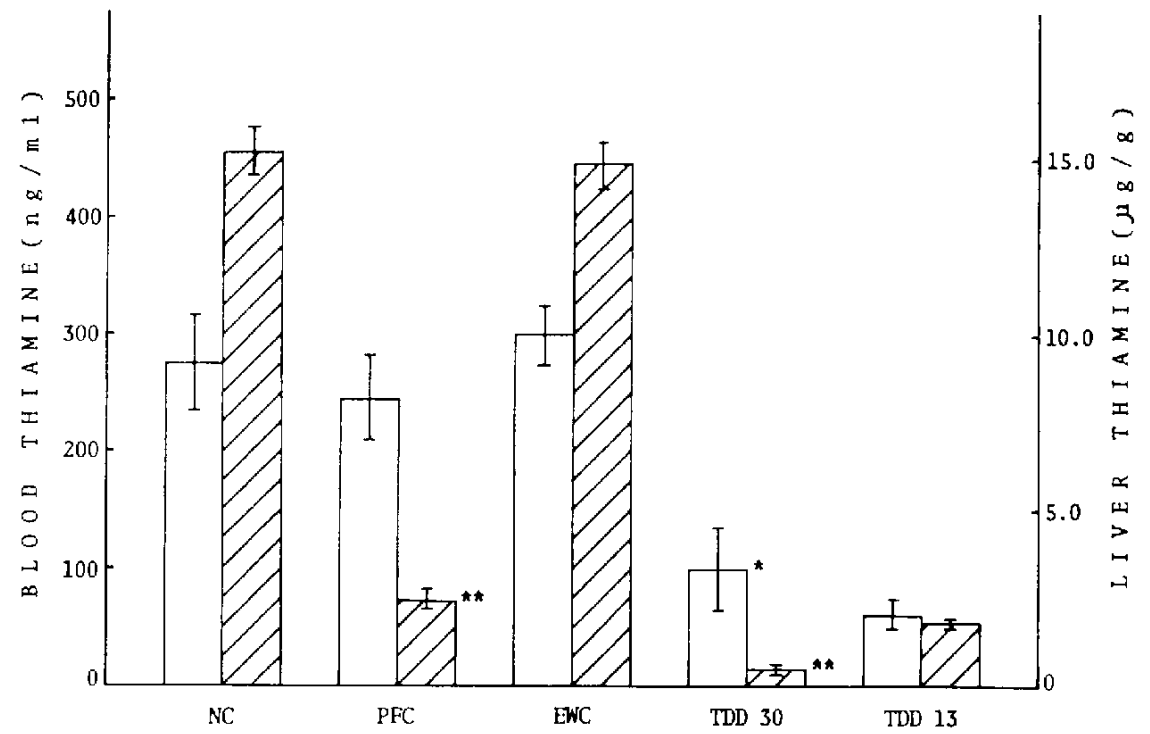

Fig. 8. Blood and liver thiamine levels. Abbreviations are the same as Fig. 2. " $p<0.01$ corresponding NC group and EWC group, " $p<0.001$ corresponding NC group and EWC group.

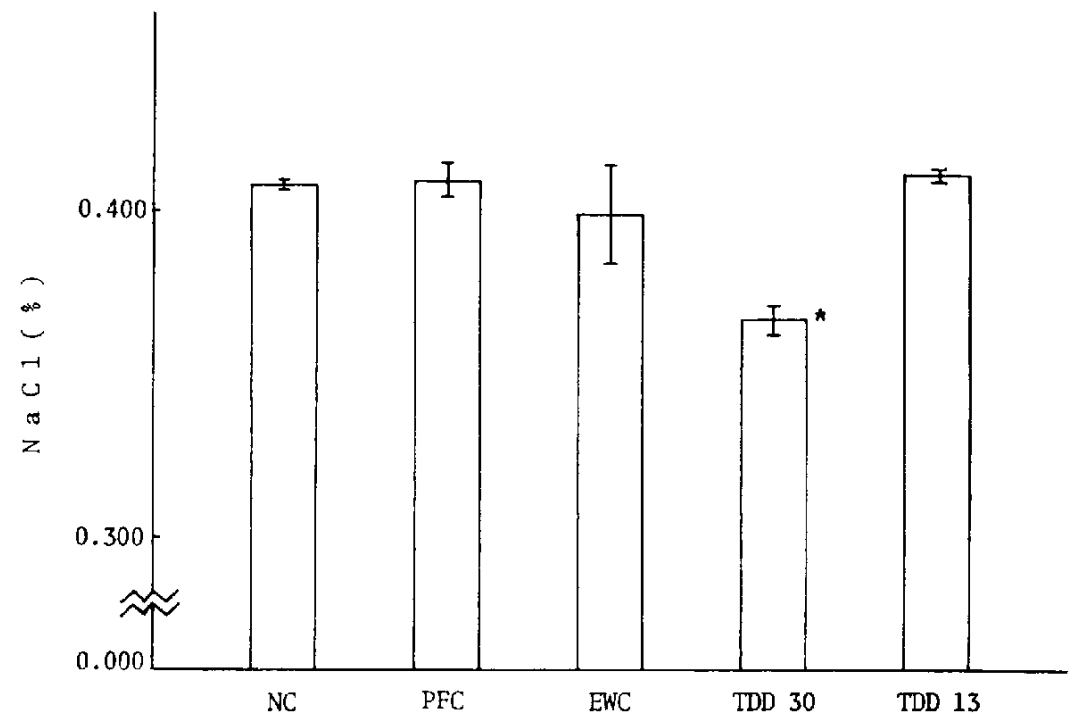

Fig. 9. Percent of $\mathrm{NaCl}$ solution, causing $70 \%$ hemolysis. Abbreviations are the same as Fig. 2. ${ }^{*} \mathrm{p}<0.001$ corresponding NC group and EWC group. 
the NC group. T levels of liver decreased to to $2.4 \mu \mathrm{g} / \mathrm{g}$ as against in the NC group $0.5 \mu \mathrm{g} / \mathrm{g}$ in the TDD30 group as against (Fig. 8).

$15.3 \mu \mathrm{g} / \mathrm{g}$ in the NC group, but the levels in

4. Properties of RBC membrane: The the EWC group were equal to that in the NC group. In the PFC group its levels decreased concentration of $\mathrm{NaCl}$ solution that causes $70 \%$ hemolysis was $0.41 \%$ in the TDD13

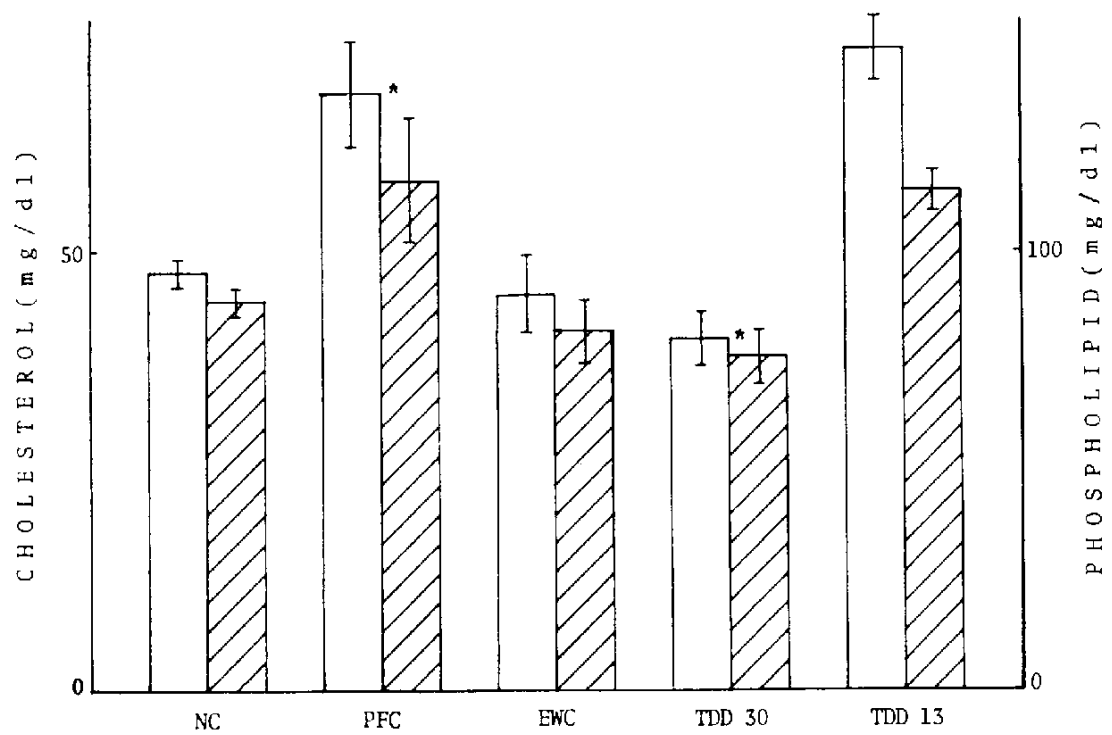

Fig. 10. Cholesterol and phospholipid levels of plasma. Abbreviations are the same as Fig. 2. ${ }^{*} p<0.02$ corresponding $\mathrm{NC}$ group.

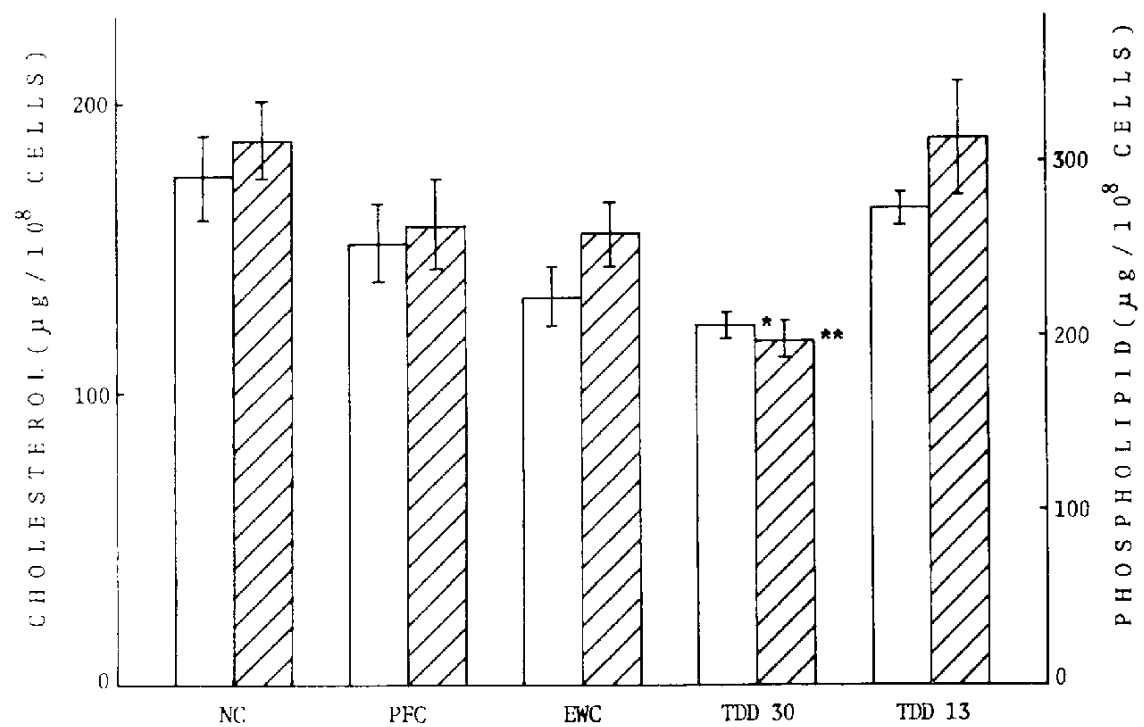

Fig. 11. Cholesterol and phospholipid levels of RBC. Abbreviations are the same as Fig. 2. " $p<0.02$ corresponding $N C$ group, " $p<0.01$ corresponding NC group and $p<0.02$ corresponding EWC group. 
group and $0.37 \%$ in the TDD30 group as against $0.41 \%$ in the NC group (Fig. 9). That is, osmotic resistance of RBC to hypotonic solution increased in the TDD30 group. but it was not changed in RBC of the TDD13 and EWC group. Cholesterol and phospholipid levels of plasma increased in the PFC and TDD13 group, but those levels decreased slightly in the TDD30 group (Fig. 10). Cholesterol and phospolipid levels of RBC membrane in the TDD30 group were $124 \mu \mathrm{g} / 10^{8}$ cells and $198 \mu \mathrm{g} / 10^{8}$ cells as against $169 \mu \mathrm{g} / 10^{8}$ cells and $312 \mu \mathrm{g} / 10^{8}$ cells in the NC group, respectively (Fig. 11). Phospholipid levels in the TDD30 group decreased significantly as against the EWC group.

\section{DISCUSSION}

Decrease in number of RBC, WBC and $\mathrm{Ht}$ values has been reported in $T$ deficient experiment in man (8). We observed the same result in rats fed a TDD for 13 days. As the body weight gain began to decrease after 10 days fed a TDD, so this stage is attributed to early stage of $T$ deficiency and may be correspond to the $T$ deficiency in man. Blood T levels in the TDD13 group were 15 to $20 \%$ of that in the NC group. Recently Yokomine et al. reported that the blood $T$ levels in man were about $68.1 \pm 31.2 \mathrm{ng} / \mathrm{ml}$ (9). Murata et al. reported that the blood T levels of boarding girls were $41 \pm 3.0 \mathrm{ng} / \mathrm{ml}$. but among them there were many girls whose $T$ levels were very low without any symptoms of beriberi (10). Their $T$ levels were 14 to $24 \%$ of the mean $T$ levels. T does not appear to be stored in the body to any appreciable extent; consequently, deficiency symptoms may be observed within a few weeks in subjects maintained on a deficient diet (11). But considering the $T$ levels of rats and humans without any symptoms, there seems to be some $T$ reserve in body to perform normal metabolism. When the blood $T$ levels decrease to about $20 \%$ or less of the mean $T$ levels, symptoms due to $T$ deficiency may be observed. On the other hand, increase in $\mathrm{RBC}, \mathrm{Ht}$ and $\mathrm{Hb}$ values were observed among the animals in the TDD30 group, and these findings were significantly different from the EWC group. Decrease in WBC may result from the chronic malnutrition. The levels of 2.3-DPG in RBC. which plays an important effect on the binding capacity of $\mathrm{Hb}$ to $\mathrm{O}_{2}$ (12), decreased markedly in the TDD30 group, and those were about $30 \%$ of that in the NC group. Decrease in 2.3-DPG causes the binding $\mathrm{Hb}$ to $\mathrm{O}_{2}$ tightly, so it is difficult for $\mathrm{Hb}$ to release $\mathrm{O}_{2}$ into peripheral tissues. The hypoxia in peripheral tissues stimulates the production of erythropoietin, so that more $\mathrm{RBC}$ are produced. Reticulocytes were not detected in the EWC group and detected infrequently in the TDD30 group, but in some cases in the TDD30 group, the number of them increases about twice of that in the $N C$ group. The blood T levels in the TDD13 group were $62 \mathrm{ng} / \mathrm{ml}$, while the levels in the TDD30 group were $102 \mathrm{ng} / \mathrm{ml}$ and very variable from 17 to $365 \mathrm{ng} / \mathrm{ml}$. The transient increase in blood $\mathrm{T}$ levels occurring at the late stage of $T$ deficiency is attributed to the mobilization of $T$ in the body to compensate the reduced RBC metabolism. The chronic reduction of glycolysis by $T$ deficiency leads to the diminution of 2,3-DPG levels. Erythrocytosis may be partly due to the decrease in 2,3-DPG levels. As blood $T$ levels in the TDD30 group, which produce erythrocytosis. were higher than that in the TDD13 group which produced anemia, $\mathrm{T}$ is an important factor for hematopoiesis. T-responsive megaloblastic anemia has been reported ( 2 . 3). The resuits that the anemia occurred at early stage of $T$ deficiency and the erythrocytosis at late stage strongly suggested that $T$ plays a key role in hematopoiesis.

$T$ levels of liver were reduced gradually as progression of $T$ deficiency in the TDD 
group. But in the PFC group $T$ was reduced to the levels of $2.4 \mu \mathrm{g} / \mathrm{g}$ at 30 days, while at 13 days the levels $(16.2 \mathrm{\mu g} / \mathrm{g})$ were the same as those in the NC group. The PFC group did not show any symptoms of $T$ deficiency, so this group was not in $T$ deficient state. At 13 days fed a TDD, the growth rate began to decrease, and the $T$ levels of liver were similar to those in the PFC group at 30 days. Liver needs $T$ to maintain its own metabolism in addition to the role of $T$ storage. So $T$ levels in the PFC group at 30 days are considered to be the minimum concentration to perform its normal metabolic functions. It is not obviously understood why the $T$ levels of liver were low in the PFC group at 30 days.

Comparing the TDD group with the PFC group, lipids levels of plasma were not changed at early stage of $T$ deficiency, but those decreased at late stage of $T$ doficiency. Ando et al. reported that osmotic resistance of $\mathrm{RBC}$ is related to the decrease in cholesterol of membrane (13). The similar phenomenon was observed in the TDD30 group, and this increased osmotic resistance to hypotonic solution was the results of the decrease in cholesterol and phospholipid of RBC membrane.

\section{REFERENCES}

1) Sauberlich, H.E.: Biochemical alterations in thiamine deficiency--Their interpretation. Am. J. Clin. Nutr. 20, 528-546 (1967)

2) Viana, M.B. and Carvalho, R.I.: Thiamineresponsive megaloblastic anemia, sonsorincural deafness, and diabetes mellitus: A new syn- drome? J. Pediat. 93, 235-238 (1978)

3) Rogers, L.E., Porter, F.S. and Didbury, J.B., Jr: Thiamine-responsive megaloblastic anemia. J. Pediat. 74, 494-504 (1969)

4) Peason, W.N.: The Vitamins VII, Edited by Bliss, C.l., Peason, W.N. and György, P. sccond edition, p. 53. Academic Press. New York (1967)

5) Bragdon, J.H.: Method for determination of total serum lipid. Lipid and the Steroid Hormones in Clinical Medicine. Editod by Sunderman. F.W., p. 9, Lippincott. Philadelphia, Montreal (1960)

6) Zak, B.: Simple rapid microtochnic for serum total cholcsterol. Am. J. Clin. Pathol. 27, 583$588(1957)$

7) Fiske, C.H. and Subbarow, Y.: The colorimetric determination of phosphorus. J. biol. Chem. $116,375-400(1325)$

8) Nishio, M., Kitamura, S. and Sahara, M.: Biochemical findings and requirement of vitamin $B_{1}$ in experimental vitamin $B_{1}$ deficiency in Japanese volunters. J. Japan. Soc. Foods Nutri. 1, 139-141(1948)

9) Yokomine, R., Kuriyama, M., Arima, H. and Igata, A.: Biochemical analyses of vitamine $B_{1}$ metabolism in normal subjects. Vitamins 52 , 89-95 (1978)

10) Murata, K., Komuro, H. and Asano, M.: Urinary and blood thiamine levels in healthy women. Vitamins 53, 1-9 (1979)

11) FAO/WHO Expert Group: Vitamin requiroments, vitamin $A$, thiamine, riboflavin and niacin. Wld. Hlth. Org. Techn. Rep. Sor. No. 362 (1967)

12) Benesch, R. and Benesch, R.E.: The effect of organic phosphates from the human orythrocyte on the allosteric properties of hemoglobin. Biochem. biophys. Res. Commun. 26, 162-167 (1967)

13) Ando, T., Yoshida, H., Terakura, T., Fukuzawa, T. and Takahashi, Y.: Osmotic resistance of crythrocytes in hepatobiliary diseases. Japan. J. Gastroenterol. 74, 329-339 (1977) 